\title{
PRINCIPI SUI FILTRI PER LE ONDE MICROBARICHE
}

\author{
Ferricicho Mosettri
}

1. - Siamo stati spinti a studiare un nuovo tipo di microlsarografo dotato di potere selettivo varialbile, per le varie frequenze, mediante filtri atti a eliminare intere bande di frequenza, per poter proseguire con maggior sicurezza e obbiettivita uno studio già iniziato ( $\left.{ }^{1}\right)$ sulla direzione di provenienza delle onde microlbariche. Si tratta di eliminare dai microbarogrammi l'effetto del vento o di altri faltori perturlbanti che, con oscillazioni a breve periodo e molto ampie mascherano completamente le onde più lunghe che maggiormente interessano. In'altra utilita che si presenla, usando tali strumenti filtranti, ¿̀ che si possono eliminare nelle stazioni reristratrici tutte le oscillazioni fuorché il ristretto gruppo di periodi che si vuol studiare, facilitando con ció di molto la stima del periodo dell'oscillazione registrata e la misura dello sfasamento presentato dai vari impulsi.

L'ingrandimento di un comune microbarografo è dato da (2)

$$
s=k \frac{K}{\sqrt{C^{2} \omega^{2}+\left(K-I \omega^{0}\right)^{\sigma}}} \frac{\omega}{\int^{2}+A^{\prime \prime}} p_{0} \sin \left(\omega t+\varphi^{\prime}\right)
$$

ove $A=\frac{\gamma P}{\rho V^{r}} \frac{\pi}{8 r_{1}} \frac{R^{4}}{1}$

$I, K, C$, rispetlivamente il momento d'inerzia, d'allrito e di richiamo del sistema oscillante,

$\gamma P$ il modulo di compressibilità adiabatico,

$\rho$ la densità del gas ed $\eta$ la sua viscosita,

$V$ il volume del recipiente,

$R$ ed 1 il raggio e la lunghezza del capillare.

Prescindendo dal primo fattore, di risonanza, la [1] ci dice che le onde di maggior periodo vengono man mano ridotte con l'aumen-

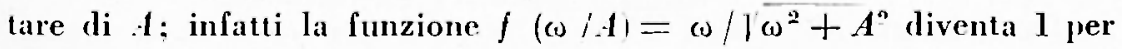
$\omega=\lambda, 0$ per $\omega=0$ ed $\dot{0}$ sempre crescente nell'intervallo $0 \leqslant \omega \leqslant \infty$, Come più sopra si diceva, molte volte, peraltro, interessereblue in- 
vece, di mantener inalterate le onde lunghe ed eliminare quelle piì brevi: per esempio le onde dovute al vento, che hanno periodo variabile da qualche secondo (o anche meno) ad un paio di minuti. Per ottenere l'eliminazione delle onde brevi abbiamo pensato di operare nella maniera seguente: la capsula registratrice venga chiusa in una scatola ermetica; essendo chiuso il capillare del recipiente di "capacità " del microbarografo, lo strumento è in condizioni di sentire solo le variazioni di pressione all'interno della scatola. Si faccia ora comunicare questa scatola con l'esterno soltanto mediante un capillare di lunghezza $l$ e raggio $r$ noti. Sia $P$ la pressione inizialmente all'interno della scatola, se $p$ è la pressione esterna variabile, si avrà, attraverso il capillare che collega la pressione interna della scatola con quella atmosferica esterna, un flusso d'aria dato dalla legge di Poiseuille:

$$
\frac{d l}{d \iota}=\frac{\pi}{8 r_{1}} \frac{r^{4}}{1}(p-P)
$$

Con procedimento analogo a quello usato nella nota $\left({ }^{2}\right)$ si oftiene. per la pressione interna alla scatola, avendo posto

$$
\begin{aligned}
& B=\frac{\gamma P}{\rho v} \frac{\pi}{8 \eta} \frac{r^{4}}{1} \\
& \frac{d P}{d t}+B P=l P
\end{aligned}
$$

lintegrale particolare che si presenta quando la pressione esterna valia con la legre $p=p_{0}$ sin (1) $t \grave{e}$

$$
P=\frac{B}{\sqrt{\left.B^{\circ}+\omega\right)^{\prime \prime}}} p_{0} \sin (\omega t+\delta) \quad \text { ove } \delta=-\operatorname{arctg} \frac{\omega}{B}
$$

Con tale modifica dovremo porre nella [1] al posto della $p_{0} \sin \left(\omega \mathrm{t}+p^{\prime}\right)$ l'espressione di $P$ testé ottenuta, per cui

$$
\left.s=l \frac{\Lambda}{\sqrt{\left(.^{2} \omega^{2}+\left(\Lambda-\overline{\left.I \omega^{2}\right)^{2}}\right.\right.}} \frac{\omega}{\sqrt{\omega^{2}+A^{\prime \prime}}} \frac{B}{\sqrt{b^{2}+\omega^{2}}} p_{0} \sin (\omega) t+\delta^{\prime}\right)
$$

la funzione $g(\omega) \quad B)=B / \sqrt{B^{-}+()^{-}} i=0$ per $(\omega)=\chi$ e diventa eguale all'unità per $(i)=0$, essa è sempre decrescente nell'intervallo $0 \leqslant \omega \leqslant \alpha$. 
Considerandola separatamente, si potranno, dunque, eliminare tutti i periodi al disotto di un periodo dato.

L'espressione

$$
s=\frac{\omega B}{\sqrt{A^{2}+\omega^{2}}} \frac{\sqrt{1}^{\prime} B^{0}+\omega^{2}}{2}
$$

parte essenziale della [4], rappresenta la curva di selettività di un filtro in cui la sensibilità per una certa frequenza può venir modificata a piacere.

Si presenta ancora il caso cbe quando si vogliono raggiungere smorzamenti troppo grandi per certi periodi, si dovrebbero usare valori di $A$ o $B$ che risultano esagerati nella pratica perché implicherebbero l'uso di capillari troppo lunghi e sottili, o di capacita troppo elevate, né sarebbe possibile mantenere il massimo ad una determinata posizione (vedi oltre), operando con $A$ e $B$ eguali, o comunque non si otterebbe mai la selettività che si può ragrgiungere, applicando, come ora mostreremo, sia al capillare del recipiente, sia a quello della scatola contenente la capsula registratrice, filtri costituiti da una o più coppie di capillari e scatole di opportune dimensioni.

Prendiamo infatti una scatola chiusa ed indeformabile di volume $v_{1}$ e facciamola comunicare con l'esterno solo attraverso un capillare $C_{1}$. Se $P_{0}$ sin $\omega t$ i la pressione esterna, per quanto si è già visto, la pressione interna alla scatola varierà con la

$$
p(t)=\frac{B_{1}}{\sqrt{B_{1}^{2}+\omega^{2}}} p_{\mathrm{o}} \sin \left(\omega t+\delta_{1}\right) \quad \text { ove }-\delta_{1}=\operatorname{arctg} \frac{\omega}{B_{1}}
$$

se adesso tale scatoletta che comunicava unicamente con l'esterno mediante il capillare $C_{1}$, la mettiamo in comunicazione anche col capillare $C$ di cui $\dot{e}$ dotata la scatola contenente il registratore, in modo insomma che quest ultimo comunichi con l'esterno attraverso il sistema costituito da "capillare + scatola + capillare ", l'equazione differenziale per la pressione agente sulla capsula registratrice sarà tenendo conto della [3] e della [6]

$$
\frac{d P}{d t}+B P=B \frac{B_{1}}{\sqrt{B_{1}^{2}+\omega^{2}}} p_{0} \sin \left(\omega t+\delta_{1}\right)
$$

da cui roi soliti procedimenti si ottiene 


$$
\left.P(t)=\frac{B}{\sqrt{B^{2}+\omega^{2}}} \frac{B_{1}}{\sqrt{B_{1}^{2}+()^{2}}} p_{0} \sin (\omega) t+\delta_{0}\right)
$$

con $\operatorname{tang} \tilde{\delta}_{2}=-\operatorname{tang}\left(\delta+\delta_{1}\right)$

Sicché operando in tale maniera la formula dellingrandimento dinamico diverreble:

$$
\left.s=k \frac{K}{\sqrt{C^{2} \omega^{2}} \overline{+\left(K-I \omega^{2}\right)^{2}}} \frac{\omega}{\sqrt{\omega^{2}} \frac{B}{+A^{2}} \sqrt{B^{2}+\omega^{2}}} \frac{\sqrt{B_{1}^{2}+\omega^{2}}}{B_{1}} P_{0} \sin (\omega) t+\delta_{3}\right)
$$

da cui risulta evidente uno smorzamento ancor magriore per le onde di più piccolo periodo. Volendo smorzare ancora di più queste onde si potrà estendere il caso grià usato ed aggiungere al sistema "scatola del registratore + capillare + scatola + capillare" ulteriori coppie "scatola +- capillare $+\ldots . . .+$ s satola ; capillare ". Se tali coppie ulteriori sono $n-1$ la pressione interna alla scatola (pressione agente sulla pagina superiore della capsulal sara del tipo:

$$
P_{\mathrm{a}}=\frac{B}{\sqrt{D^{2}+\omega^{2}}}\left(\frac{B}{\sqrt{B_{1}^{2}+\omega^{2}}}\right)^{n} P_{0} \sin \left((1) t+\delta_{\mathrm{n}}\right)
$$

ove $p=p_{0} \sin \omega t \dot{t}^{2}$ la pressione esterna.

Si vede cosi come, fino ad una cerla frequenza (1) „. si posia, non solo. ridurre l'ampiezza di unionda qualunque. di quanto si vuole, ma che si possa anzi ridurre sempre più l'ampiezza sia delle onde più brevi che delle onde più lunghe. Come già si disse la $s$ ci rappresenta in tal maniera una tipica curva di filtro che ha la massima permeabiliti per un certo periodo. Aumentando il valore dell'esponente n diminuisce anche l'ampiezza del massimo della curva. però sempre molto di meno che non l'ampiezza delle falde, di destra e, sopratlutto, di sinistra. II massimo, in questo caso, non si sposta mai, mantenendosi sempre fissato in corrispondenza ad una cerla ascissa di frequenza $\left(\omega_{0}\right.$, .

Estendendo alle onde di magrior periodo i ragionamenti svolti riguardo l'estinzione dei periodi più piccoli, si potrà ottenere di aumentare, analogamente al caso precedente, anche lo smorzamento di queste onde, e cioè, mantenendo fissato il massimo, abbassare la falda di destra della curva di selettivitì [5].

Supponiamo di avere una certa scatoletta di volume $v_{2,}$ munita di un capillare $c_{2}$, dotata di una capsula virfuale; per quanto si è delto in [2], lo spostamento di questa capsula virtuale sarà, prescindendo dalla risonanza meccanica 


$$
S_{02}=H \frac{\therefore}{\sqrt{\left(1^{2}+d_{2}^{2}\right.}} p_{3}
$$

Se si unisce tale capsula virtuale col pressistente capillare del recipiente di sensilyiliti in maniera che anche tale recipiente, come gria la scatola contenente la capsula registratrice, commichi con l'esterno anziché direttamente, attaverso il sistema, "capillare + scatola t capillare", lo spostamento del registratore per il solo effetto di questi elementi diverrà

$$
s=I I \frac{(1)}{\sqrt{(1)^{2}+A^{*}}} \frac{\omega}{\sqrt{(1)^{2}+A_{2}^{2}}} p_{0} \sin \left((1) t+\varphi_{1}\right)
$$

proseguendo nell'analogia col caso precedente e supponendo di fornire il recipiente di sensililita di un sistema di m coppie " capillare t scatola " lo spostamento della capsula sarà, sempre non considerando per ora, l'effetto dellaltro sistema di filtri agente sulla scatola ermetica

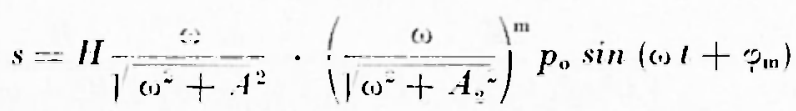

In definitiva, quando l'apparato registratore foapsula idropneumatical sia chiuso " e-ternamente" in una scatola comunicante con la pressione atmosferica da misurare solo attraverso un capillare e un sistema di $n$ coppic "satola t- capillare" tutti eguali fra di loro, ed "internamente" comunichi con un recipiente (di sensililità) il quale $a$ pure csiso in comunicazione con lesterno attraverso un capillare ed un analogo sistema di $m$ elementi "scatola + capillare " purc equali, si avà una registrazione selettiva. di selettivita tanto maqriore quanto piǹ elevati sono i numeri $n$ ed $m$. del tipo

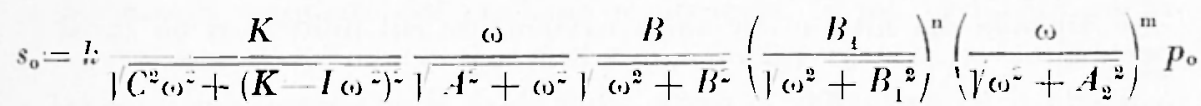

Varra a tal punto far notare che se uno dei capillari che mettono in comunicazione con lesterno la capsula, c̀ tale che $B=0$ o $B_{1}=0$ (e quindi dalla $\mid 9] s_{o}=0$ ); nessunonda può venir messa in evidenza. Se invece a chiuso il rapillare del recipiente o uno dei cipillari del filtro del recipiente, allora $i t=1)$ o $A_{2}=0$ e lo morzamento delle 
onde lunghe viene alterato; anzi, poiché praticamente se un capillare i-esimo $\dot{e}$ chiuso, $\dot{e}$ come se tutti gli $i$ capillari fossero chiusi, tutti i rari $\frac{\omega}{\sqrt{\omega^{2}+p^{2}}}$ diventano 1 e non esiste più alcun smorzamento per le onde lunghe.

Cosi non esiste smorzamento per le onde brevi se tutti i capillari esterni hanno aperture eccessivamente elevate tanto che i $B$ siano $>>$ ( ) e le varie $B / \sqrt{B^{*}+\omega^{*}} \sim 1$ (se un solo filtro presenta elevata apertura del capillare è come se non esistesse). Ancora, se tutti i capillari del recipiente di sensibilita (capillari interni) sono troppo aperti allora $\omega / \sqrt{\omega^{*}+A^{\circ}} \sim 0$ e nessuna onda viene messa in evidenza $1 *$ \%

I limiti per i valori di $A$ e $B$ che si vogliono usare, e quindi i limiti costruttivi delle parti variabili del filtro - nel nostro caso l'apertura del capillare - si possono ottenere facilmente in base alla frequenza limite che si vuole sia possibile registrare.

La [9] si può scomporre in due gruppi di fattori:

$$
\text { Nella } S=k \frac{K}{\sqrt{C^{2} \omega^{2}+\left(K-I \omega^{2}\right)^{2}}} \frac{\omega}{l^{\prime} \omega^{2}+A^{2}} \frac{B}{\sqrt{R^{2}+\omega^{2}}}
$$

che dà l'ingrandimento e la selettività del microbarografo, dotato sia del recipiente di sensibilità (secondo fattore) che di una scatola contenente la capsula (terzo fattore), che della capsula stessa (primo fattore), e che, una volta costruito lo strumento con queste particolarita rimane fissata e costante;

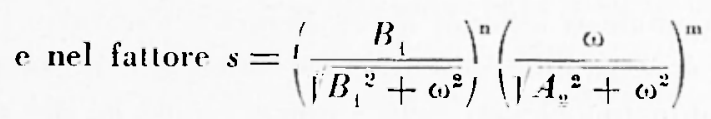

che dipende dai filtri e che varia ovviamente col numero $n$ od $m$ di essi.

$\left.{ }^{*}\right)$ Poiché $A$ e $B$ dipendono oltre che dal raggio del rapillare anche dalla lunglieza di questo e dal volume della raparita romnessa, le medesime ronsiderazioni fatte sullapertura del rapiliare, si potrebbero fare anche sulla lunglieza di questo o sul volume della raparita; si considera solo lapertura del rapillare perché, volendo onerare ron $A$ e $B$ ralcolatamente variabili, $i$, in pratica, molto piì facile far variare l'apertura del eapillare, the non le altre parti del filtro. 
Studieremo ora come si modificano le curve $S$ o $s$ al variare dei parametri che le definiscono.

Per cercare di individuare la forma della curva $s$, poiché aumentando o facendo comunque variare $n$ od $m$ si ha il solo risultato di diminuire con una certa legge l'ampiezza della curva, basterà per ora studiare la curva di sensibilita ridotta:

$$
s_{0}=\frac{B}{\sqrt{\omega^{2}+B^{2}}} \frac{\omega}{\sqrt{\omega^{2}+A^{2}}}
$$

Meritera fermarsi su i due casi distinti $A=B$ e $A \neq B$. Nel primo caso la curva ha un massimo per $B=\omega$ e l'ordinata di tale massimo ha il valore $B^{2} / 2 B^{2}=1 / 2$. Potremo dunque mettere in risalto una certa frequenza $\omega_{0}$, nel caso $B=A$, facendo si che le costanti valvolari delle coppie dei filtri siano tali che

$$
\frac{\gamma^{P}}{\rho} \frac{\pi}{8 \eta} \frac{r^{4}}{l V}=\omega_{0}
$$

cioce ancora, tenendo conto dei valori normali c.g.s.,

$$
\omega_{\mathrm{o}}=2,4 \cdot 10^{9} \frac{r^{4}}{l I^{\prime}}
$$

Qualora si voglia aumentare la selettivita (sia pure a scapito dellingrandimento - male questo del resto facilmente ovviabile -) per la frequenza $\omega_{1,}$, hasterà aumentare l'ordine della curva assegnando agrli esponenti $n$ ed $m$ valori (interi) maggiori di 1 . Osserveremo che se si aumenta $n$ più di $m$ si avrà uno smorzamento maggiore delle frequenze a sinistra del massimo e viceversa, in tali casi però anche il massimo si sposta (vedi avanti); quando invece $n$ ed $m$ aumentano egualmente, entrambe le falde della curva si abbassano ed il massimo rimane fisso.

Considerando invece la curva s con $A \neq=B$, il suo massimo risulta per (1) $=\sqrt{A B}$, si puo anche rua legare mumricamente la frequenza da esaltare con le costanti delle coppie filtranti mediante la

$$
\omega_{0}=2.4 \cdot 10^{\circ} \sqrt{\frac{r_{\mathrm{A}} r_{\mathrm{B}}{ }^{4}}{l_{\mathrm{A}} l_{\mathrm{B}} V_{\mathrm{A}} I_{\mathrm{B}}}}
$$


lordinata corrispondente a questo massimo vale

$$
\frac{1}{1+\frac{A}{B}}
$$

Tale modo di operare può alle volte esser di pregio poiché si può rendere l'ordinata del massimo anche maggiore di I/2 dell'ampiezza reale dell'onda selezionata (come invece si ottiene per $A=B$ ), infatti :

se $A<B$ sarà sempre $f / B<1$ e quindi

$$
\frac{1}{1+\frac{A}{B}}>1 / 2
$$

Ee invece $A>B$ sarà sempre $A / B>1$ e quindi

$$
\frac{1}{1+\frac{A}{B}}<1 / 2
$$

Inoltre, in tal maniera, dipendono $\omega_{0}$ dai due parametri $A$ e $B$, per ogni $B$ fissata vi sarà un 4 tale che $\omega_{0}=\sqrt{413}$ e viceversa, esisteramno dunque infinite possiloilità di soluzione.

Anche in questo caso aumentando $n \circ m$ si oltiene un corrispondente aumento della selettività.

Comunque sia, riepilogando, perché venga filtrata una certa frequenza $\omega_{0}$ bastera che sia

$$
B=(1)_{0}
$$

oppure

$$
\sqrt{A B}=(1)_{0}
$$

a seconda che sia $A=B$ oppure $A$ diverso da $B$.

Il grado di selezione potrà poi regolarsi fentro i limiti della sensibilita dello strumentof facendo opportunamente crescere i valori di $n$ e di $m$.

Quando $m=n$ il rapporto di ogni ordinala di una curva di ordine $n$ con l'ordinata corrispondente della curva di ordine $n-1$ rimane costante, il valore di questo rapporto varia pero da ascissa a ascissa. Per qualsivoglia ascissa, (nel caso $A=B, n=m$ ) il valore 
del massimo è $1 / 2$ per $n=1,1 / 4$ per $n=2 \ldots \ldots 1 / 2^{n}$ per $n=n$. Aumentando l'ordine della curva il massimo risulta sempre più spiccato rispetto all'ordinata delle altre frequenze. Per esempio se, essendo $A=B=0,024, \mathrm{e} m=n$ assume successivamente i valori $], 2,3,4,5,6$, i rapporti successivi tra l'ordinata del massimo (che cade per il pe-

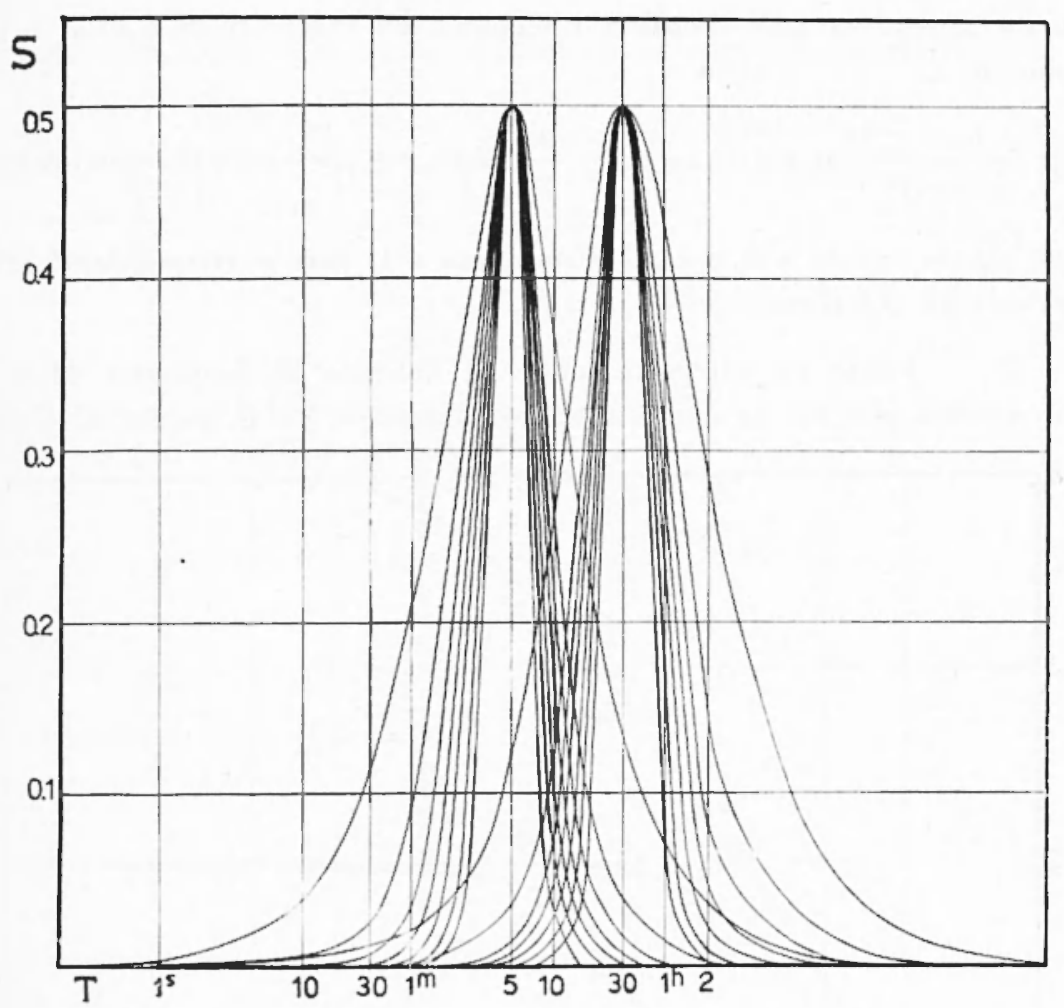

Fig. 1. Due tipiche curve di selettiviti $s^{\mathrm{mm}}=f^{\mathrm{m}} \mathrm{s}^{\mathrm{m}}$ per lond: di 5 min e per londa di 30 min, con $t=B$ e $m=n$, per $m=1,2,3,4.5 .6,7$. Le ampiezze delle curve con $m>1$ vemero moltiplicale rispettivamente per $2,4,8 \ldots$ - in modo da lat sciar inalterata loordinata del massino.

riodo di $4,4^{\text {min }}$ ) e quella del periodo di $1^{\text {min }}$ assume $i$ successivi valori 2,$1 ; 4,4 ; 9,3 ; 19,5 ; 39,0 ; 87,0 ;$ dai quali si vede quanto possa aumentare la selettiviti della curva aumentando l'ordine $n$ di essa. Basandosi su quanto è stato precedentemente svolto, al)hiamo calcolato alcune curve di sensililiti per alcune frequenze, come si può vedere nella figura 1 . 
Mediante l'applicazione dei filtri si ottiene in definitiva che la funzione

$$
y=\sum_{i}^{\mathrm{n}} a_{\mathrm{i}} \sin \left(\omega_{\mathrm{i}} t+\varphi_{\mathrm{i}}\right)
$$

rappresentante l'oscillazione naturale di pressione viene trasformata in un'espressione più semplice, a seconda del valore di $B$ o di $A$ e $B$, secondo la

$\sum_{i}^{n}\left(\frac{\omega_{\mathrm{i}} B}{\omega_{\mathrm{i}}{ }^{2}+B^{2}}\right)^{\mathrm{P}} a_{\mathrm{i}} \sin \left(\omega_{\mathrm{i}} t+\varphi_{\mathrm{i}}\right)=\frac{a_{\mathrm{o}}}{2^{\mathrm{p}}} \sin \left(\omega_{0} t+\varphi_{\mathrm{o}}\right)+$ termini trascurabili

ove $\omega_{0}$, sia equale a $B$ e $a_{0}$, 仓̧o l'ampiezza e la fase corrispondenti alla periodiciti selezionata.

2. - Esiste un ulteriore modo di definire la frequenza filtrata (frequenza per cui la curva $s$ ha un massimo) ed è quello di tener

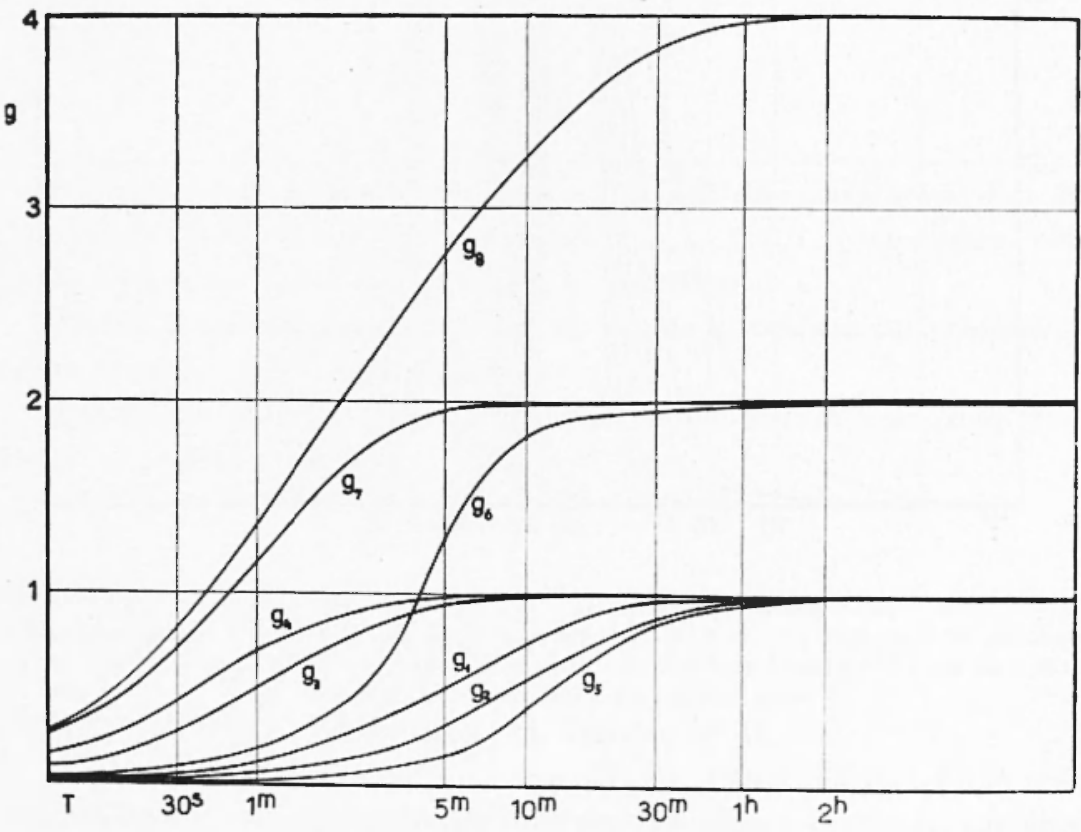

Fi⿺. 2 - Valori assunti dalle curve $g=\frac{B}{\sqrt{6^{2}+1}-2}$ semplici e combinate, in funzione del periodo, per vari valori di $B$. Curve semplici: $g_{1}$ ron $B=0,012$; $g_{4}$ con $B=$ $=11.1167: g_{: 1}$ con $\left.B=0,160\right): g_{1}$ con $B=0.600$. Curve combinate: $g$; combinazione delle quattro $g$, qualora $\mathrm{i}$ filtri corrispondenti siano posti in serie $\left(g_{5}=g_{1} \times g_{z} \times\right.$ $\left.\times y: \times g_{1}\right) ; g_{0:}=g_{1}+g$. (in parallelol; $g_{0}=g_{: l}+g_{1}$ (in parallelo); g. combinazione delle quattro $g$. con filtri in parallelo $\left(g_{2}=g_{1}+g_{2}+g_{3}+g_{1}\right)$. 
conto anche di esponenti $m$ ed $n$ diversi fra di loro. In questo caso, che è il più generale, si ha

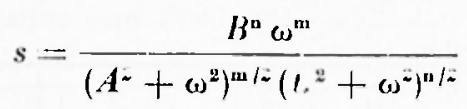

procedendo come al solito si ha, per unica soluzione possibile

$$
\omega_{0}=\sqrt{\frac{\left(1-\frac{n}{m}\right) 1^{2}}{2 \frac{n}{m}}+\frac{\sqrt{\left(1-\frac{n}{m}\right)^{2} A^{4}+4 A^{2} B^{2} \frac{n}{m}}}{2 \frac{n}{m}}}
$$

se $n=m,(1),=\overline{A B}$ come già si vide.

Nel caso più semplice che sia $B=A \grave{e}$

$$
s_{n}=\frac{B^{n} \omega^{\mathrm{m}}}{\left(\therefore+\omega^{2}\right)^{\frac{m+n}{2}}}
$$

il massimo is dato allora dalla

$$
\omega_{0}=B \sqrt{\frac{n}{m}}
$$

esso varia in ampiezza e si sposta come si vuole a seconda dei valori di $n$ ed $m$.

Dalla $\mid 13]$, quando $m$ is equale a $n$, qualunque sia il loro valore, $\left(\omega_{1},=B\right.$ come wià i vide precedentemente.

In tutti questi casi testè esaminati, il registratore è posto " in serie " attraverso due pile più o meno lunghe di coppie "scatola + capillare" con la pressione esterna, si ottiene cosi come risultato, di selezionare una banda più o meno stretta, che al limite può diventare una linea, dello spettro delle onde di pressione.

3. - $\mathrm{Si}$ possono evidentemente disporre anche più pile di coppie "scatola + capillare" anziché in "serie", "in parallelo" fra lo strumento o l'esterno, sia soltanto in modo che la scatola disponga 
di questi filtri in parallelo sia che soltanto il recipiente ne disponga, sia ancora che entrambi ne dispongano.

La disposizione in "parallelo" si ottiene facendo comunicarc,

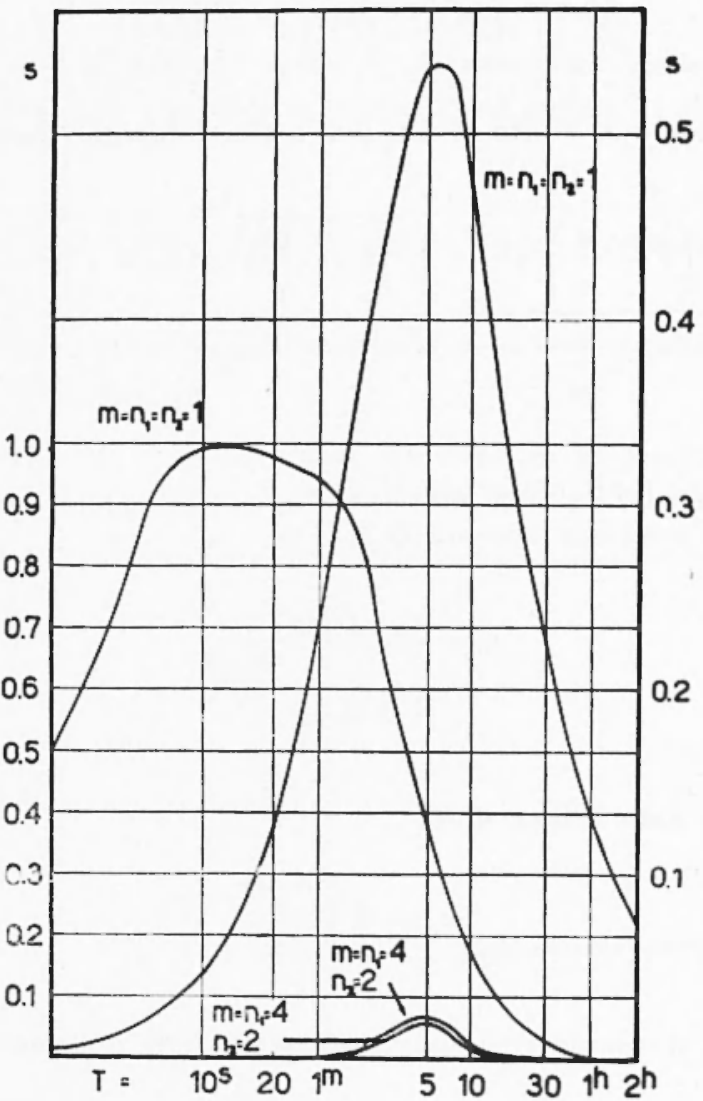

Fig. 3 - Alcune curve di selettiviti $s=f_{1}\left(g_{1}+g_{\sharp}\right)-$ (ioè a dire con due coppie in parallelo al registratore e una coppia al recipiente ottenute con disposizioni particolari delle coppie filtranti. La corva a sinistra (cui corrisponde la scala di sinistral è lia $s=f_{1}\left(g_{1}+g_{:}\right)$con $A=0,\left(124, B_{1}=0,0028 i, B_{4}=6,28\right.$. La curval di destria è dello stesso tipo e venne ottenuta ron $A=B_{1}=0.02 \mathrm{t}$ e $B_{:}=0,00287$. Le due pircole curve inferiori col massimo sulla stessa ascissa del massimo della curva precedente, differiscono da questa solo per i parametri $m, n_{1}, n$.

una o l'altra o entrambe le parti dello strumento con l'esterno, anziché mediante un solo sistema di filtri, attraverso due o più sistemi. $\grave{\mathbf{E}}$ facile vedere che, in tal caso, per csempio quando solo la scatola sia posta in parallelo con l'esterno, attraverso due o più filtri, 
due o più gruppi diversi di impulsi di pressione più o meno alterati in ampiezza e fase si sommano sulla capsula, mentre quando le coppie sono disposte in serie, gli elfetti si moltiplicano. Se per esempio la scatola comunica con l'esterno attraverso due sistemi di coppie

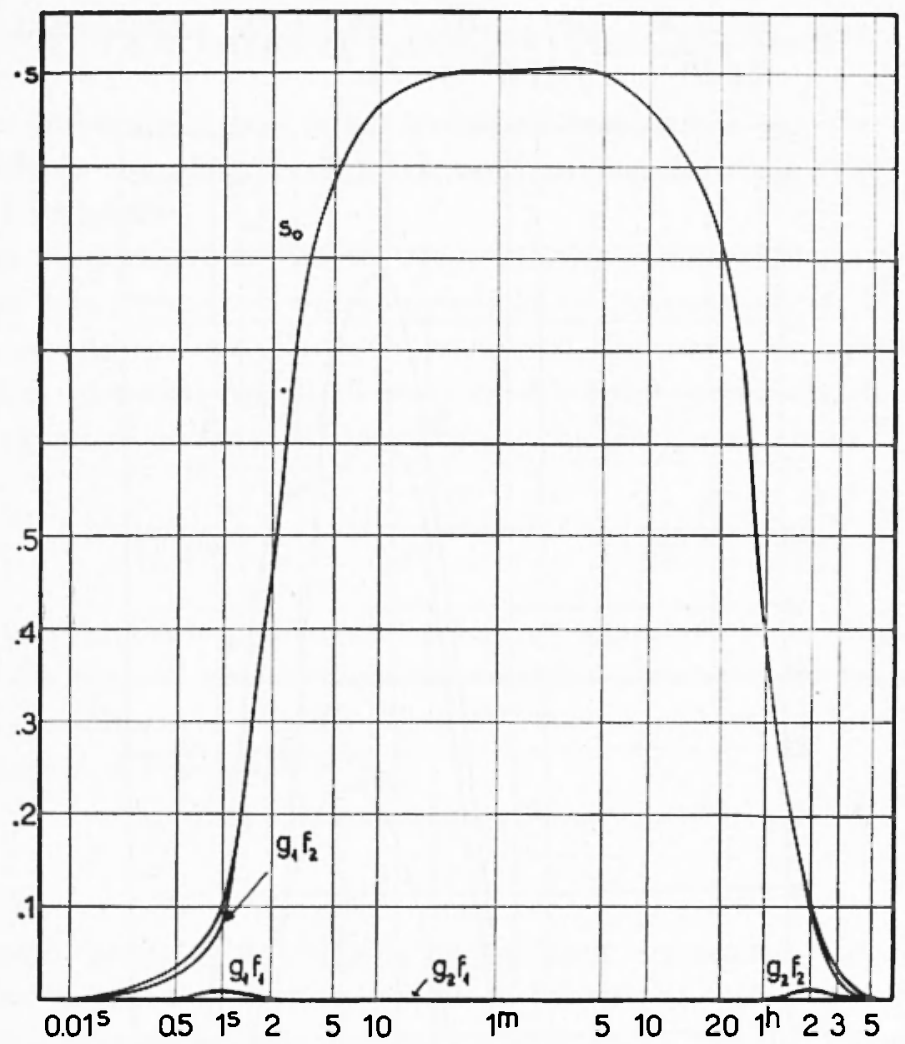

Fig. + - Curve di selettiviti ottenute con due gruppi di sette coppie filtranti eguali poste in parallelo sul registratore e altrettunte sul recipiente; - curve cioè del tipo

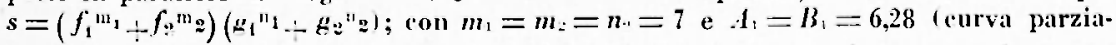
le $g_{1} f_{1}$ con massimo ner 1 sec) $A_{2}=B_{2}=0,00088$ (curva $g_{:} f_{2}$ con massimo per $T=2$ ore).

di filtri una composta di $p$ elementi eguali di parametro $B$, e l'altra di q elementi equali di parametro $B^{\prime}$, la pressione agente sulla capsula, traseurando l'effetto del recipiente, sarì, se $p_{w}=p_{n}$ sin (1) $l$ è la pressione esterna

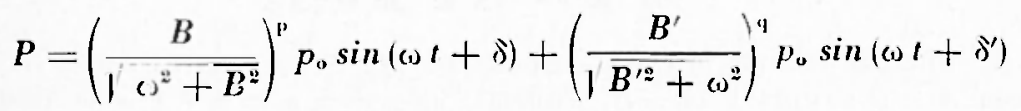


da cui si vede che per opportune differenze di fase si può avere anche interferenza, sia pure solo parziale perché le ampiezze non sono eguali (si tratta visibilmente di un caso di sorgenti incoerenti).

Se invece i due sistemi di filtri fossero disposti successivamente uno all'altro (in "serie ") si avrebbe

$$
\left.P=\left(\frac{B}{\sqrt{B^{2}+\omega^{2}}}\right)^{p}\left(\frac{B^{\prime}}{\sqrt{B^{\prime \prime}+\omega^{2}}}\right)^{3} \Gamma_{0} \sin (\omega) l+\delta_{1}\right)
$$

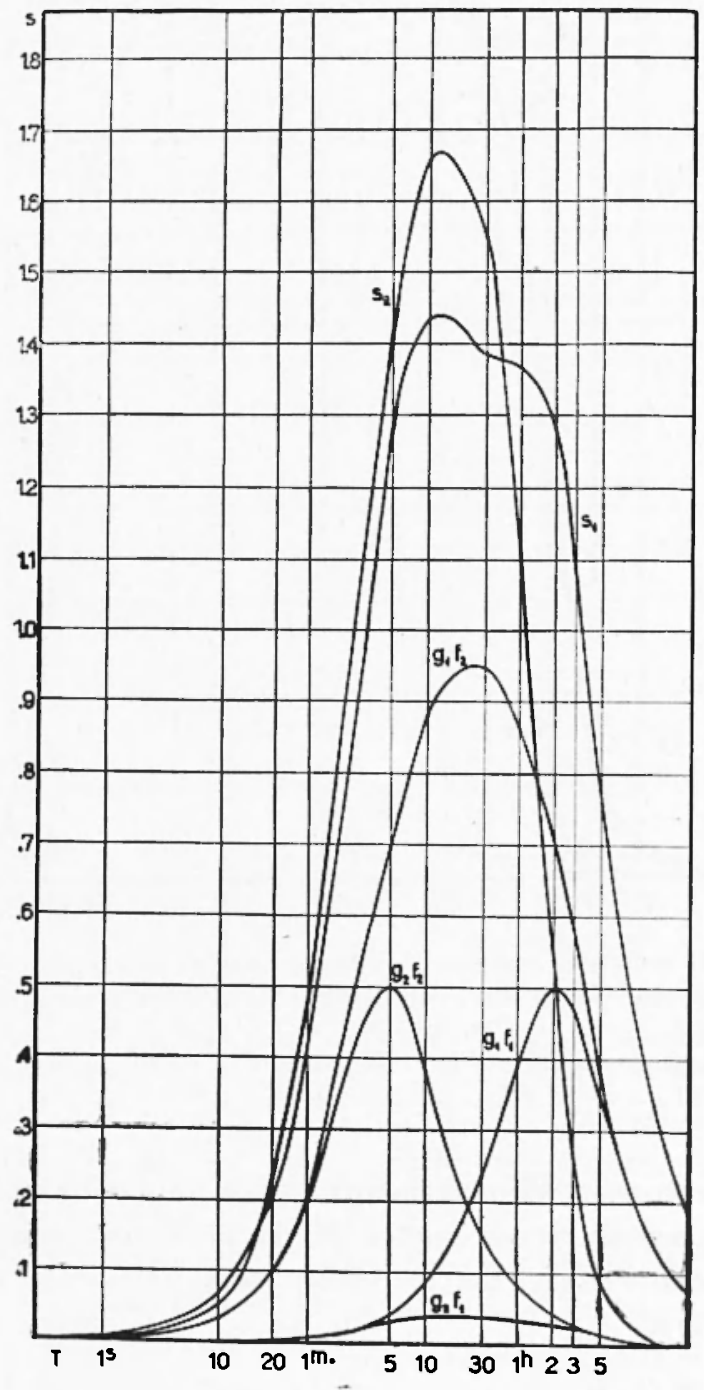

Fig. 5 - Ulteriore esempio di curve composte $s=\left(11^{m}+12^{2} 2\right)\left(2^{n_{1}}+{ }^{\prime} \cdot 2^{\mathrm{n}} 2\right)$, $s_{1}$ con $A_{1}=B_{1}=0,02 \cdot 4$ e $A:=B .1=0,0028 \pi, m_{1}=m_{2}=n_{i}=n \cdot+1$ e le relative componenti; $s_{2}$ con $A_{1}=B_{1}=0,00088$ e $A_{2}=B_{1}=0,0209$. 
nella fig. 2 mostriamo un esempio di un caso del genere ottenuto con quattro filtri posti in serie $o$ in parallelo; le curve $g_{1}, g_{2}, g_{3}, g_{4}$ rappresentano contributi di ogni filtro, la $g_{\text {; }}$ dà un esempio dell'effetto filtrante che si ottiene quando i quattro filtri sono posti in serie llordine in cui essi sono posti non ha alcuna importanza), la $g_{8}$ indica invece l'effetto che si ottiene ponendo questi quattro filtri in parallelo.

Naturalmente è ben più interessante considerare ciò che succede per l'effetto complessivo di filtri, posti in parallelo sia sulla scatola che sul recipiente.

Per esempio, il recipiente di sensibilità comunichi con l'esterno con una sola serie di $m$ coppie eguali la cui costante sia $A$, la scatola invece comunichi con l'esterno attraverso due serie in "parallelo" una di $n_{1}$ clementi eguali di costante $B^{\prime}$, l'altra composta di $n_{2}$ elementi eguali di costante $B^{\prime \prime}$ la curva di sensibilità avrà allora la forma

$$
s_{\mathrm{o}}=\left(\frac{\omega}{\sqrt{A^{\prime 2}+\omega^{2}}}\right)^{\mathrm{m}}\left[\left(\frac{B^{\prime}}{\sqrt{B^{\prime 2}+\omega^{\prime}}}\right)^{\prime \prime}+\left(\frac{B^{\prime \prime}}{\left(\sqrt{B^{\prime \prime}+\omega^{2}}\right)^{\mathrm{n}}}{ }^{2}\right]\right.
$$

in tal caso i massimi assumono posizioni ottenibili meno immediat:mente che nei casi precedentemente esaminati, per esempio nella eventualità elementare in cui $A=B^{\prime}=B^{\prime \prime}, m=n_{1}=1, n_{2}=2$, si ha che la frequenza del massimo è

$$
\omega_{10}=\sqrt[1]{\frac{2 A^{2}+A^{3}}{A^{2}+4 A-2}}
$$

Casi come questo si potrebbero moltiplicare e generalizzare. Comunque, ora, le curve possono assumere le forme più disparate e diverse, possono essere ristrette e diventare molto ampie oppure possono venire allargate di quanto si vuole in modo che il filtro non lasci più passare una sola riga dello spettro, ma una banda più o meno larga. tutto questo senza tener conto dello sfasamento ma basandosi soltanto sulla deformazione dell'ampiezza. Alcuni esempi in merito sono mostrati nella fig. 3 .

Il caso dei filtri in "parallelo" può venir generalizzato. Per esempio, se oltre che la scatola anche il recipiente di sensibilità comunica con due serie di elementi eguali, in numero rispettivamente di $m_{1}$ e $m_{2}$, con costanti $A^{\prime}$ e $A^{\prime \prime}$, la curva di selettivita $s$ arrà la forma

$$
s=\left[\left(\frac{\omega}{\sqrt{A^{\prime 2}+\omega^{2}}}\right)^{\mathrm{m}}{ }_{1}+\left(\frac{\omega}{\sqrt{A^{\prime \prime 2}+\omega^{2}}}\right)^{\mathrm{m}}=2\right]\left[\left(\frac{B^{\prime}}{\sqrt{B^{\prime \prime \prime}+\omega^{2}}}\right)^{\mathrm{n}}+\left(\frac{B^{\prime \prime}}{1 B^{\prime \prime 2}+\omega^{2}}\right)^{\mathrm{n}}=\right]
$$


esempi di particolari curve di questo tipo sono rappresentati nelle figg. 4 e 5 in cui sono raffigurate anche le componenti parziali della s, date dai prodotti del I termine per il III, del II per il III, ece. A seconda del valore delle costanti $A$ e $B$ e degli esponenti $m$ ed $n$ il con. tributo di tali componenti può essere più o meno elevato.

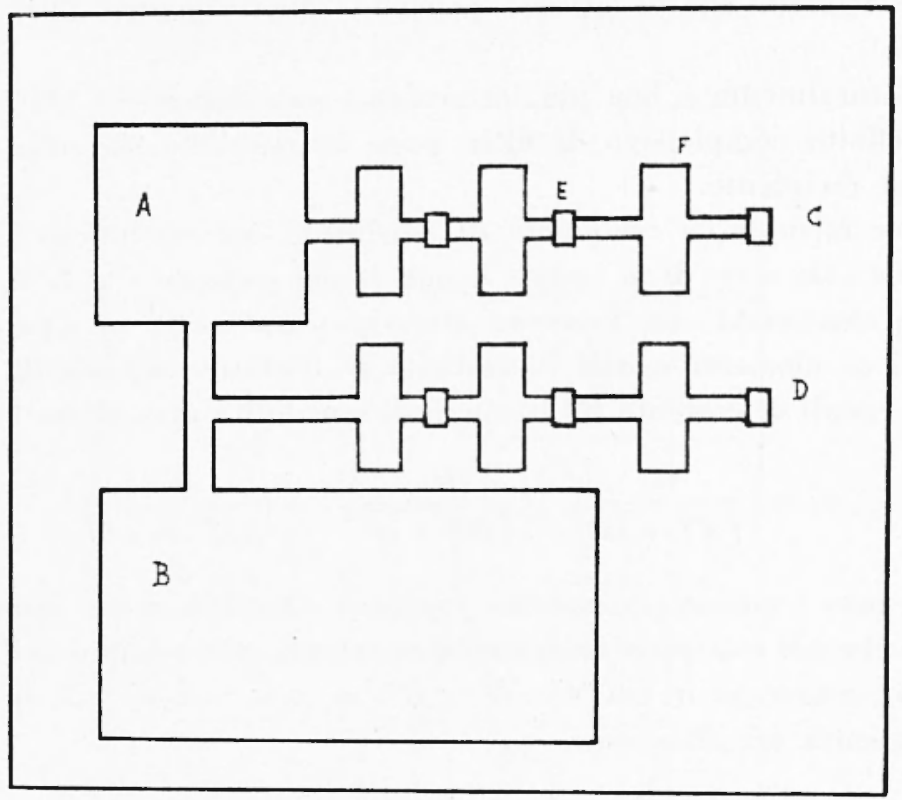

Fig. 6 - Schema di principio del nuovo microbarografo: A registratore con scatola ermetica; B recipiente di capacità; $C$ filtri per le onde brevi; D filtri per le onde lunghe; E capillare graduato: F scatoletta di capacita.

Ancor più generalmente, quando si abbiano in parallelo $v$ filtri nella scatola e $s$ filtri nel recipiente, riadottando $\mathrm{i}$ simboli $g_{1} \mathrm{p}^{\mathrm{p}}\left(\omega / B_{1}\right)=$ $\left(\frac{B_{1}}{\sqrt{\left.B_{1}{ }^{2}+\omega\right)^{2}}}\right)^{p}$ e $f_{1}^{9}\left(\omega / A_{1}\right)=\left(\frac{\omega}{\sqrt{\left(\omega^{2}+A_{1}^{2}\right.}}\right)^{9}$ si ha per la curva di seletti. vita la forma:

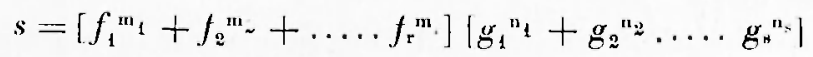

in tal modo operando, si ottiene come sopra, ma con maggiori possibilità, di modificare come si vuole la forma della curva.

Cercare però col calcolo, in tali condizioni, la posizione dei massimi della funzione, e cioè la banda di frequenza per cui si ha selettività, è molto spesso difficile e non sempre possibile. 
Gia volendo cercare tali punti nella semplicissima

$$
s=\left(\frac{\omega}{\sqrt{A^{2}+\omega^{2}}}\right)^{m}\left[\left(\frac{B^{\prime}}{\sqrt{B^{\prime 2}+\omega^{2}}}\right)^{\prime \prime}+\left(\frac{B^{\prime \prime}}{\sqrt{B^{\prime \prime 2}+()^{2}}}\right)^{\mathrm{n}}\right]
$$

si dovreblie risolvere l'equazione

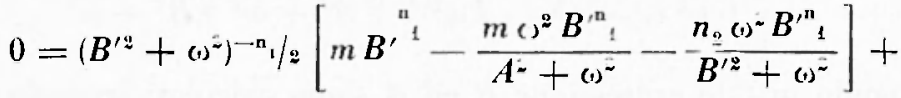

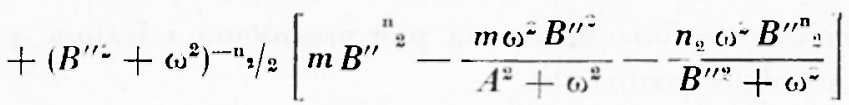

che è appunto solubile rispetto ad $\omega$ solo per taluni valori di $m_{1}$ ed $n$..

In tutte queste disposizioni arrehle anche importanza la conoscenza delle fasi che per ora ahhiamo trascurato ma che possono influire anche parecehio sulla forma della curva.

Le stesse formule principali $\omega_{0}=B$ e $\dot{\omega}_{0}=\mid B .4$ che servono per i casi più semplici ed usuali dei filtri (filtri eguali in serie), servono anche per conoscere la forma della curva di selettivita risultante

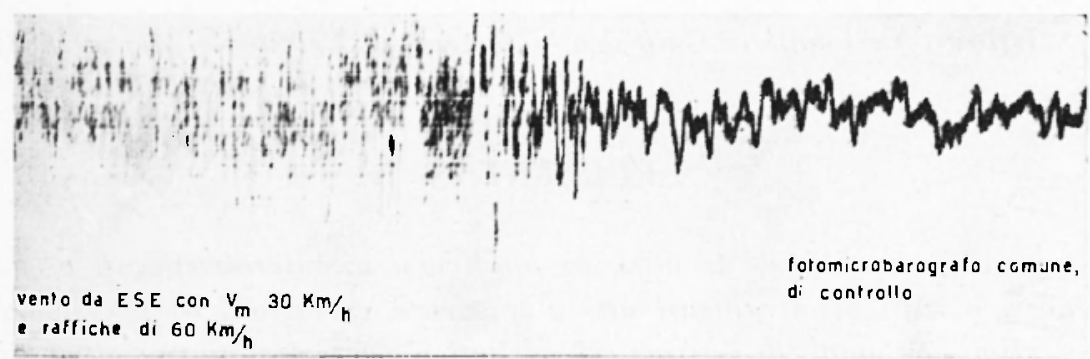

nuovo Polomicrobarogralo
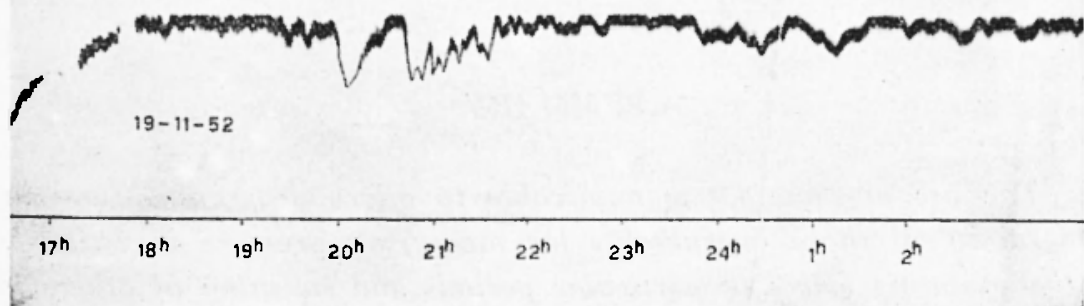

Fig. 7 - Esempio di registrazione con i filtri pes leliminazione delle onde di piecolo periodo: leffetu del rento $i$ quasi completamente annullato. nel grafico ottenuto rol nuovo strumenlo. 
dal primo elemento filtrante: lo strumento registratore stesso, costituito, come si sa, dalla scatola ermetica con capillare contenente la capsula registratrice e dal recipiente di sensibilità, strumento il cui fumzionamento $\dot{e}$ retto dalla formula

$$
S=h \frac{K}{\sqrt{\iota^{\circ} \omega^{2}+\left(K-I \omega^{2}\right)^{2}}} \frac{\omega}{\sqrt{A^{2}+\omega^{2}}} \frac{B}{\sqrt{B^{2}+\omega^{2}}}
$$

Quando in tale espressione $B$ ed $A$ siano calcolati secondo le modalità già esposte, una prima sia pur grossolana selezione puó venir operata grià dallo strumento.

Concludendo, con il metodo da noi descritto ed adottato si riesce ad esälare, selezionandole, onde di qualsivogrlia periodo senza incorrere in alcuna delle difficolta pratiche che si incontrerebbero qualora si volesserc ottenerc i medesimi risultati cercando di esaltare certe frequenze sia direttamente mediante fenomeni di risonanza meccanici, sia traducendo gli impulsi di pressione in impulsi elettrici ed operando con la risonanza elettrica. Sarebbe infatti ben difficile costruire un oscillatore meccanico o elettrico che risuoni, per esempio, con i periodi di due ore che usualmente capitano ai microbarografi!

Istituto Nazionale di Geofisica - Osserv. di Trieste - Marzo 195.3.

\section{RIASSUNTO}

Si discute sulluso di fltri da applicarsi ai microbarografi $e$, in genere, a tutti gli strumenti atti a misurare variazioni di pressione, per eliminare onde di particolari periodi e per selezionarne altre. Si danno le equazioni delle principali curve di selettivitì dei filtri o si insegna il loro impiego.

\section{SUMMARY}

The use of some filters applicable to microbarograples, (and in general to all those instruments for measuring pressure variations), for eliminating waves of particular periods and selection of others is discussed.

Equations of the principal curves of the selectivity of the filters and instructions for their use are given. 


\section{BIBLIOGRAFIA}

(1) F. Mosettı: Primi risultati sulla localizzazione delle perturbazioni atmosferiche dallo esame delle onde microbariche. Annali di Geofisica, n. 4, 1953.

(-) F. Mosetti: Teoria del microbarograjo Allani. Annali di Geofisica, n. I, 1951. 